\section{Case Reports in Ophthalmology}

\title{
Alternating Hypotropia with Pseudoptosis: A New Phenotype of Congenital Cranial Dysinnervation Disorder
}

\author{
Fady Sedarous $^{\mathrm{a}}$ Toby Y.B. Chan ${ }^{\mathrm{b}}$ Inas Makar \\ ${ }^{a}$ University of Toronto, Toronto, ON, Canada; ${ }^{b}$ McMaster University, Waterloo Regional \\ Campus, Kitchener, ON, Canada; ' $I v e y$ Eye Institute, University of Western Ontario, \\ London, ON, Canada
}

\section{Keywords}

Congenital cranial dysinnervation disorders · Cranial nerve miswiring · Ocular synkinesis

\begin{abstract}
Congenital cranial dysinnervation disorders, also known as CCDDs, are characterized by aberrant innervation to extraocular and facial muscles resulting in unusual forms of incomitant strabismus. Anomalous innervation to extraocular muscles can result in a wide variety of phenotypes causing various clinical conditions such as Duane syndrome, congenital fibrosis of the extraocular muscles, and Möbius syndrome. We report a case of bilateral dysinnervation disorder causing atypical ocular movements in both eyes as the patient changes fixation from one eye to the other and from right gaze to left gaze that fits with the wider diagnosis of CCDDs.

\section{Introduction}

Congenital cranial dysinnervation disorders, also known as CCDDs, are characterized by aberrant innervation to extraocular and facial muscles resulting in unusual forms of incomitant strabismus. Anomalous innervation to extraocular muscles can result in a wide variety 
of phenotypes causing various clinical conditions such as Duane syndrome, congenital fibrosis of the extraocular muscles, and Möbius syndrome. We report a case of bilateral dysinnervation disorder causing atypical ocular movements in both eyes as the patient changes fixation from one eye to the other and from right gaze to left gaze that fits with the wider diagnosis of CCDDs.

\section{Case Report}

An 18-year-old female was referred for management of alternating ptosis associated with strabismus. According to the patient history, her ocular deviation had been present since birth and had not changed over the years. She had never experienced diplopia. There was no family history of any ocular motility abnormalities. Her perinatal history and past medical history were also unremarkable.

On examination, the patient was able to spontaneously alternate fixation in the primary position. While fixating with the right eye, the left eye showed moderate exotropia and as the right eye moved inwards towards left gaze, there was increasing left hypotropia and ptosis (Fig. 1). When the patient fixated with the right eye in right gaze, there was significant limitation of left adduction and upshoot of the left eye (Fig. 1). Similarly, when the patient was fixating with the left eye, the right eye showed moderate exotropia and on attempted left adduction the right eye showed exotropia and significant hypotropia and ptosis (Fig. 2).

The ocular alignment varied widely with change of fixation and with any slight change in the direction of gaze (Fig. 3). On examining ocular rotations monocularly, there was significant limitation of ductions of the left eye more than the right eye. Left eye ductions demonstrated no adduction, limited elevation and depression, while the right eye showed mild limitation of adduction only. There was no evidence of globe retraction on ocular movement. Attempted forced duction test during the clinic examination was not possible due to very limited patient cooperation. Her visual acuity was $20 / 20$ in both eyes. Pupil examination showed equal and symmetrical pupils, with no change in pupil size during ocular movements. Examination of the anterior and posterior segment was unremarkable for both eyes.

\section{Discussion}

We believe that our patient's clinical picture fits within the wide spectrum of ocular abnormalities described as CCDDs caused by aberrant axonal wiring as suggested by Nugent et al. [1], but her specific phenotype has not been published before.

The main abnormality is present in the primary position with alternating exotropia, hypotropia, and ptosis when the patient fixates with either eye. The ocular alignment varied widely with spontaneous change of fixation from one eye to the other and the ocular misalignment changed significantly with any slight change in the direction of gaze. As the patient fixates with the right eye, we notice left exotropia and as the right eye moves towards adduction looking in left gaze, we notice significant left hypotropia and ptosis. Similarly, as the patient fixates with the left eye, we notice right exotropia and on attempted left adduction in right gaze, we notice significant right hypotropia and ptosis. In right gaze, the right eye fully abducts while the left eye shows no adduction as expected but rather exaggerated supraduction and in left gaze, there is mild limitation of right adduction and significant left hypotropia with ptosis. 
This unusual clinical presentation does not fit with the typical picture of left third cranial nerve palsy associated with aberrant regeneration often described in the literature secondary to trauma or tumors, and has also been reported in congenital third CNP [2]. Several findings go against this diagnosis. As our patient fixates with the left eye, her right eye shows hypotropia and ptosis instead of the expected right hypertropia caused by extra innervation of the yoke muscles needed to bring the left eye to primary position per Hering's law of equal innervation. If our patient had left hypotropia secondary to partial 3rd CNP, she should demonstrate hypotropia in the primary position as well as left and right gaze, rather than only in left gaze. The presence of bilateral ocular involvement leads us to believe that these findings cannot be explained by isolated partial oculomotor nerve palsy.

We hypothesize that the main pathology could localize to the interneurons arising from the 6th $\mathrm{CN}$ nucleus. These neurons normally cross the midline and connect via the contralateral medial longitudinal fasciculus to the contralateral medial rectus subnucleus. As our patient looks in right gaze, the left eye elevates rather than adducts, which could be attributed to neuronal miswiring connecting the right 6th $\mathrm{CN}$ nucleus to the left superior rectus subnucleus instead. Similarly, as the patient looks in left gaze, there is abnormal connection between the left 6th $\mathrm{CN}$ nucleus and the right medial rectus muscle. Horizontal gaze palsy with progressive scoliosis is another classic example of such anomalous ocular movements thought to be caused by uncrossed or absent oculomotor and abducens internuclear tracts [1].

Currently, CCDD is the term given to conditions with congenital strabismus associated with abnormal development of cranial nerve nuclei and/or axonal innervation [3-5]. Ocular synkinesis accompanies various CCDDs $[3,6,7]$. These conditions are often secondary to mutations in genes that are crucial in the development and connectivity of cranial nerve nuclei. At least 9 genes and 26 phenotypes are currently recognized for CCDDs [8].

Other abnormal ocular movements that could not be explained by isolated cranial nerve palsies have been reported in the literature and attributed to CCDDs, including children presenting with ptosis on adduction (bilateral or unilateral) [6, 9]. Khan [6] described a similar case of supraduction during attempted adduction and infraduction during attempted abduction in the left eye. His patient also had exotropia in the primary position, but unlike our patient the contralateral eye was normal. The clinical picture of our patient suggests an abnormality in the 6th and 3rd cranial nerve nuclei as well as the connecting internuclear neurons causing abnormal innervation of the EOMs. This patient demonstrates a new phenotype of the CCDDs that adds to all other described phenotypes. Imaging with high-resolution MRI would have been useful in giving us more details on the 6th and 3rd cranial nerves and their nuclei; unfortunately, this patient was lost to follow-up, hence further investigations such as high-resolution MRI and genetics testing was not possible.

\section{Statement of Ethics}

Our patient has given her informed consent regarding the publication of the material.

\section{Disclosure Statement}

The authors have no financial disclosures. All coauthors certify that the submission is original work, which has not been submitted for publication elsewhere. 


\section{Case Reports in Ophthalmology}

\section{References}

-1 Nugent AA, Kolpak AL, Engle EC: Human disorders of axon guidance. Curr Opin Neurobiol 2012;22:837-843.

-2 Freedman HL, Kushner BJ: Congenital ocular aberrant innervation - new concepts. J Pediatr Ophthalmol Strabismus 1997;34:10-16.

-3 Engle EC: Oculomotility disorders arising from disruptions in brainstem motor neuron development. Arch Neurol 2007;64:633-637.

-4 Oystreck DT, Engle EC, Bosley TM: Recent progress in understanding congenital cranial dysinnervation disorders. J Neuroophthalmol 2011;31:69-77.

-5 Assaf DD: Congenital innervation dysgenesis syndrome (CID)/congenital cranial dysinnervation disorders (CCDDs). Eye 2011;25:1251-1261.

-6 Khan AO: A novel form of aberrant innervation in congenital cranial dysinnervation disorder. J AAPOS 2009;13:105-106.

7 Engle EC: Human genetic disorders of axon guidance. Cold Spring Harb Perspect Biol 2010;2:a001784.

-8 Bosley TM, Abu-Amero KK, Oystreck DT: Congenital cranial dysinnervation disordes: a concept in evolution. Curr Opin Ophthalmol 2013;24:398-406.

-9 Mendes S, Beselga D, Campos S, Neves A, Campos J, Carvalho S, Silva E, Castro Sousa JP: Possible rare congenital dysinnervation disorder: congenital ptosis associated with adduction. Strabismus 2015;23:33-35.

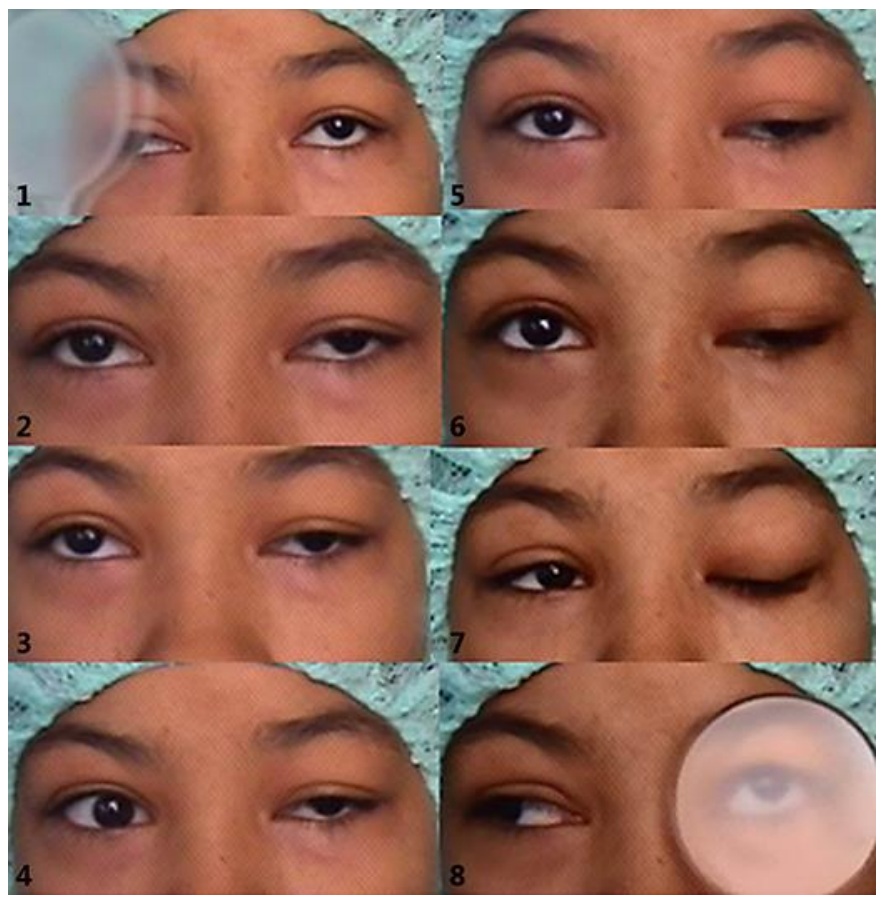

Fig. 1. 1, fixing left eye moderate RXT; 2 , fixing right eye moderate LXT; 3 , fixing right eye more LXT + slight hypotropia + left ptosis; 4, fixing right eye (slight adduction) LXT + slight left hypotropia + left ptosis; 5-7, fixing right eye further in left gaze significant left hypotropia and ptosis; 8, fixing right eye in abduction + left eye upshot. 
Case Reports in
Ophthalmology

Case Rep Ophthalmol 2018;9:96-101 DOI: 10.1159/000485832

(c) 2018 The Author(s). Published by S. Karger AG, Basel www.karger.com/cop

Sedarous et al.: Alternating Hypotropia with Pseudoptosis: A New Phenotype of Congenital Cranial Dysinnervation Disorder

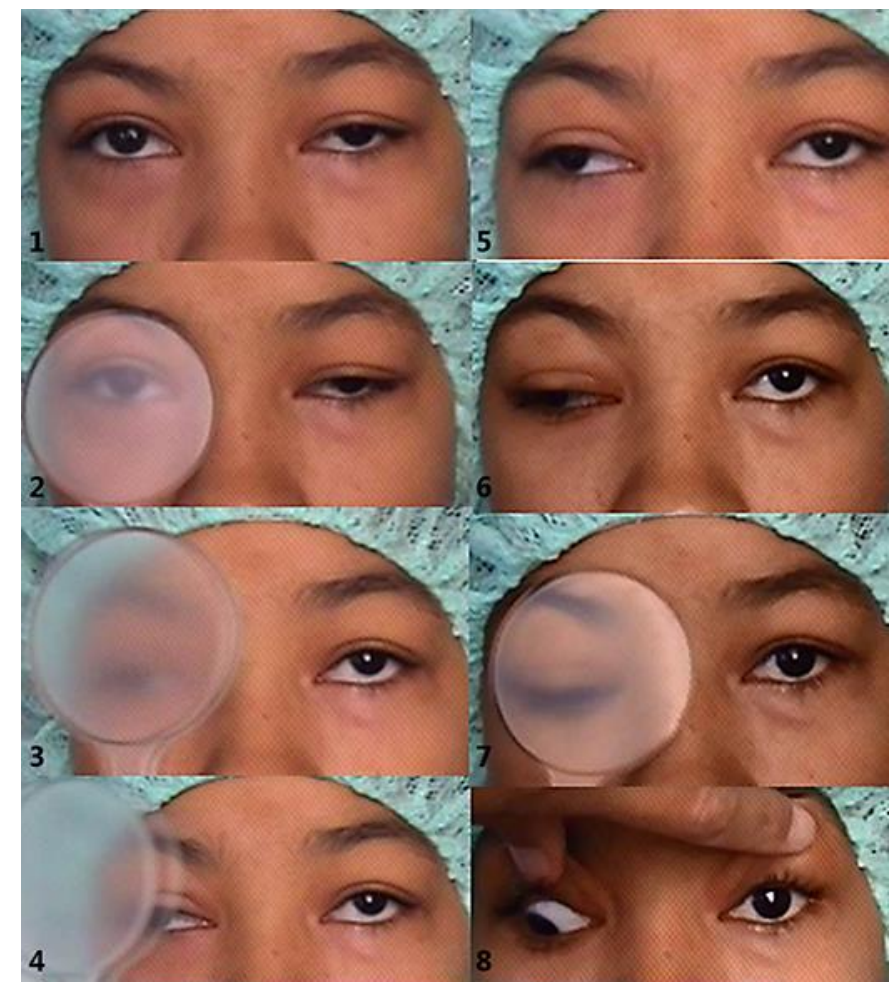

Fig. 2. 1, fixing right eye left XT + ptosis; 2 , occluding RE; 3-5, fixing left eye RXT + slight right hpotropia + slight right ptosis; 6,7 , fixing left eye in primary position + significant right hypotropia; 8, fixing left eye in attempted dextrodepression + significant right hypotropia. 


\section{Case Reports in Ophthalmology}

\begin{tabular}{l|l}
\hline Case Rep Ophthalmol 2018;9:96-101 \\
\hline DOI: 10.1159/000485832 & $\begin{array}{l}\text { @ 2018 The Author(s). Published by S. Karger AG, Basel } \\
\text { www.karger.com/cop }\end{array}$ \\
\hline
\end{tabular}

Sedarous et al.: Alternating Hypotropia with Pseudoptosis: A New Phenotype of Congenital Cranial Dysinnervation Disorder

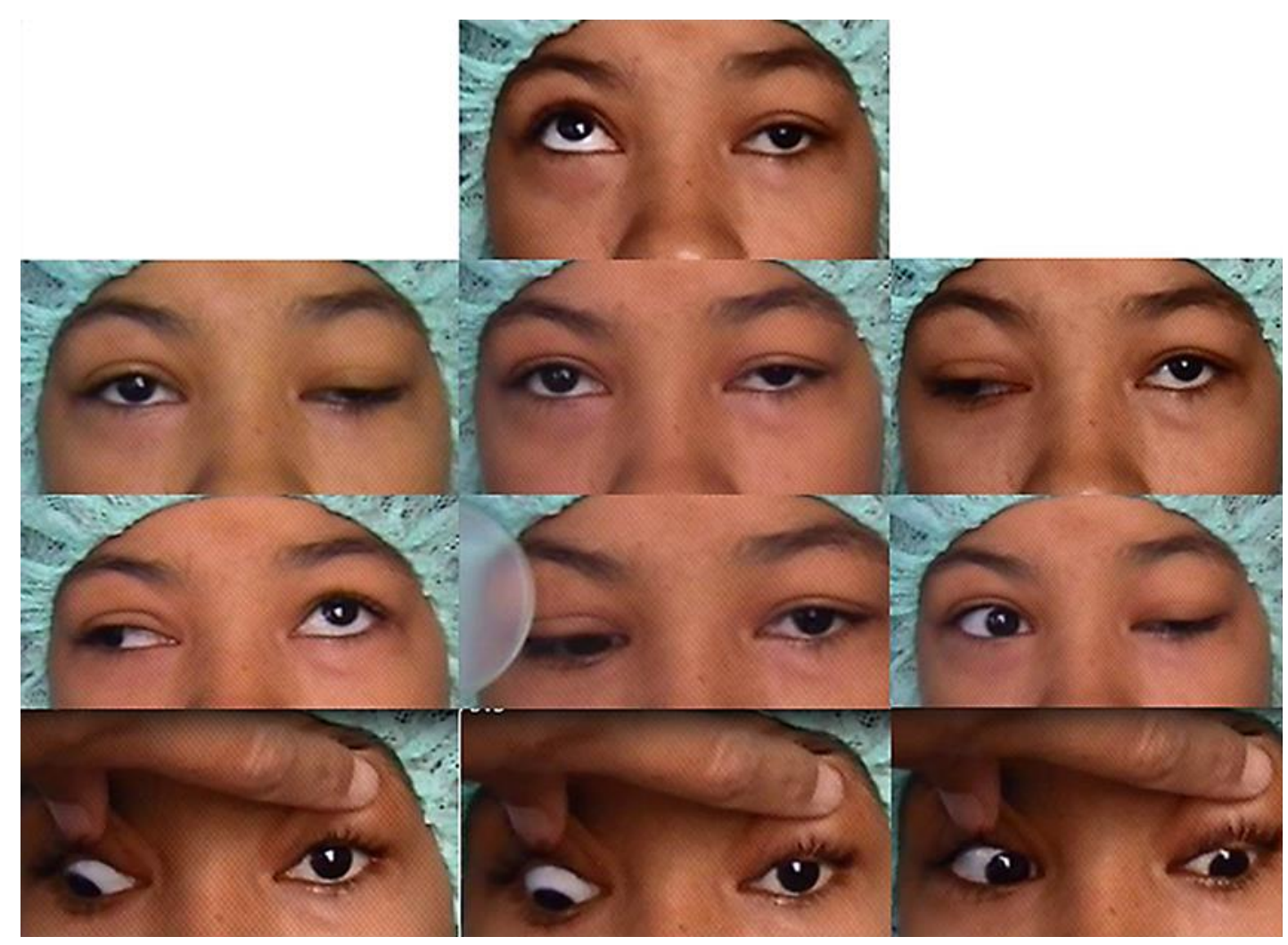

Fig. 3. Ocular rotations in different gaze positions. 